needed a blood transfusion; the rest responded to "ferrivenin." Six of the seven ultimately had normal confinements at home. The other went into labour while in hospital and had a normal confinement there. All other cases responded to oral iron.

\section{Abnormalities of Labour}

A reasonably light space and sufficient table room for instruments are regarded as safety factors in the conduct of labour. A sterile drum containing cap, mask, gown, gloves, and instruments for suturing forms part of the equipment. This is re-sterilized as necessary by kind co-operation of the local hospital. A sterile intravenous giving-set, together with dextran or plasma, is also considered essential.

Of the 94 cases eventually confined at home complications of labour occurred in four: one of foetal distress (a primigravida), one of uterine inertia (a para-14), and two cases of post-partum haemorrhage. One case of post-partum haemorrhage was slight, and was controlled by ergometrine. The other three cases were admitted to hospital (Table II, Cases 18-20)

Because of lack of facilities operative procedure is avoided in the home whenever possible. An appendicectomy on the kitchen table might well be fraught with less danger and complications than a difficult forceps upstairs. Where the hospital is reasonably close, transport does not usually present great difficulties.

Two cases were transferred to hospital in labour. It was felt that the primigravida with foetal distress (Case 18) might need forceps in order to save the baby. It was also possible that the baby might have needed resuscitation measures requiring facilities not available in the home. Fortunately these were unnecessary. The baby was born spontaneously in hospital after a comparatively short second stage.

In Case 19 the os was dilated about two fingerbreadths. The patient had vague pains off and on, and had never properly started. She was tired and anxious. Although originally she could not be persuaded to book with the hospital, she needed little persuasion at this stage.

Case 20 had a sudden post-partum haemorrhage of 3 pints (1.7 litres) after the placenta had been delivered. She collapsed and her blood pressure fell to 80/?. Ergometrine controlled the haemorrhage and intravenous dextran was started immediately. Her condition improved enough to enable her to be transported to hospital ; the drip was kept going on the way. Her condition rapidly improved in hospital following blood transfusion.

Post-partum haemorrhage is unforeseeable. It is the greatest enemy of all concerned with domiciliary midwifery. Of the accepted methods of prevention and treatment, estimation of the haemoglobin during the antenatal period, the routine administration of intramuscular ergometrine with hyalase to the mother at the time of delivery of the anterior shoulder of the infant, and the rapid administration of intravenous therapy are the most powerful weapons we possess in reducing the incidence and minimizing its effects. The patient may then be enabled to survive long enough until blood and other life-saving measures become available, or restored sufficiently to enable transport to hospital to be undertaken in safety. If blood is sent for cross-matching as soon as the diagnosis is made, valuable time, perhaps a life, may be saved.

\section{Summary}

The following points are considered important in achieving a high degree of safety in domiciliary midwifery : suitable home conditions; a system of antenatal care involving the use of a record chart and close cooperation with the midwives : a strict selection of cases for home confinement ; avoidance where possible of all operative procedures in the home ; and special measures for preventing and dealing with post-partum haemorrhage.

\section{EXCRETION OF MECAMYLAMINE AFTER INTRAVENOUS AND ORAL ADMINISTRATION}

\author{
BY \\ K. D. ALLANBY, M.D., M.R.C.P. \\ AND \\ J. R. TROUNCE, M.D., M.R.C.P. \\ From the Department of Medicine, Guy's Hospital, \\ London
}

Ganglion-blocking compounds have been widely used in the treatment of hypertension since the introduction of hexamethonium, and in many cases satisfactory control of the blood pressure can be obtained with one of these substances alone, although the best results are probably achieved in combination with the rauwolfia alkaloids. Their use has greatly improved the prognosis in malignant and non-malignant hypertension (Doyle and Smirk, 1955 ; McMichael and Murphy, 1955; McQueen and Smirk, 1956). Although entirely effective when administered parenterally, hexamethonium is often unsatisfactory when used orally. Harington (1953) showed that hexamethonium was poorly absorbed from the intestine, only $5 \%$ or less of the dose being recovered from the urine, and its effect was short-lived, thus making frequent large doses a necessity. Furthermore, its absorption is greatly influenced by the taking of food, and increasing amounts may be absorbed as intestinal stasis occurs, leading to bouts of unpredictable hypotension. For these reasons oral hexamethonium has been replaced by pentolinium and chlorisondamine chloride, though pentolinium itself is not free from similar drawbacks when employed orally.

Freis (1955) and Ford, Madison, and Moyer (1956) have reported that mecamylamine ("inversine"), a substance with ganglion-blocking properties, is well absorbed from the alimentary tract. Mecamylamine is 3-methylaminoisocamphane and is a secondary amine, a type of compound not hitherto used for its ganglionblocking effect. Encouraging reports of its use have already been published by Doyle, Murphy, and Neilson (1956) in this country, in addition to those of Freis and of Ford $\dot{e} t$ al. in the United States.

The present report is concerned with the excretion of mecamylamine, following intravenous and oral administration, in hypertensive subjects without renal failure.

\section{Methods}

All subjects investigated were hospital in-patients suffering from essential hypertension. In addition to a complete history and physical examination, a 6-foot $(1.8 \mathrm{~m}$.) chest radiograph and a 12-lead electrocardiogram were obtained in all patients. . Renal function was assessed by estimation of the blood urea and by concentration and dilution tests: no patient showed impaired renal function as judged by these methods.

Before administration of mecamylamine, each subject had been at rest in bed for at least one week, without any treatment apart from mild sedation. The blood pressure was measured lying and standing at least four times daily in all patients.

The method described by Brodie and Udenfriend (1945) was used to estimate the urinary concentration of mecamylamine. The drug is extracted from alkalinized 
urine by shaking with ethylene dichloride, and when mixed with methyl orange forms a chromagen which may be measured colorimetrically. It was found that infected urines might give false results, and to prevent bacterial growth all specimens were treated with $5 \%$ thymol and stored at $4^{\circ} \mathrm{C}$.

Since normal urines contain small amounts of a substance which forms a similar chromagen with methyl orange (usually equivalent to $50-100 \mu \mathrm{g}$. mecamylamine per $100 \mathrm{ml}$.) it is necessary to allow for this in patients receiving mecamylamine. The nature of this substance was not determined, but it appeared to be excreted at a constant rate, and the amount excreted per 24 hours varied little in each individual. The total excretion of the non-specific chromagen in 24 hours was determined in each patient before giving mecamylamine, and the appropriate fraction was subtracted from individual estimations.

Urinary $p \mathrm{H}$ was estimated with Gurr's universal indicator.

\section{Urinary Secretion after Single Oral and Intravenous Doses}

Fig. 1 illustrates the urinary excretion of mecamylamine after single oral and intravenous doses in three subjects. During the first 24 hours the amount recovered was approxi-
The fall in blood pressure may last up to 12 or more hours and the duration of effect appears to be related largely to the speed of excretion. Figs. 1 and 2 furthermore show that mecamylamine is excreted slowly in subjects with normal renal function, and that only $50 \%$ or less of the total amount excreted after a single oral dose is recovered in the first 12 hours. This agrees with the clinical observations of Ford et al. (1956), Doyle et al. (1956), and Smirk and McQueen (1957) regarding the duration of hypotensive effect after mecamylamine.

\section{Effect of Urinary $p H$ upon Excretion of Mecamylamine}

Baer et al. (1956) have demonstrated the remarkable effect of urinary $p \mathrm{H}$ on the renal clearance of mecamylamine in dogs. They found that the drug was freely secreted by the renal tubules when the urine was acid, but that reabsorption occurred if the urine became alkaline.

Fig. 3 shows the effect of urinary $p \mathrm{H}$ upon the excretion of mecamylamine. Alkaline urines were produced by giving $2 \mathrm{~g}$. (30 gr.) each of sodium bicarbonate and potassium citrate four-hourly, and $2 \mathrm{~g}$. (30 gr.) of ammonium chloride four-hourly was used to ensure an acid urine. It will be seen that the excretion of mecamylamine is extremely low in an alkaline urine, but that over $50 \%$ of an oral dose is
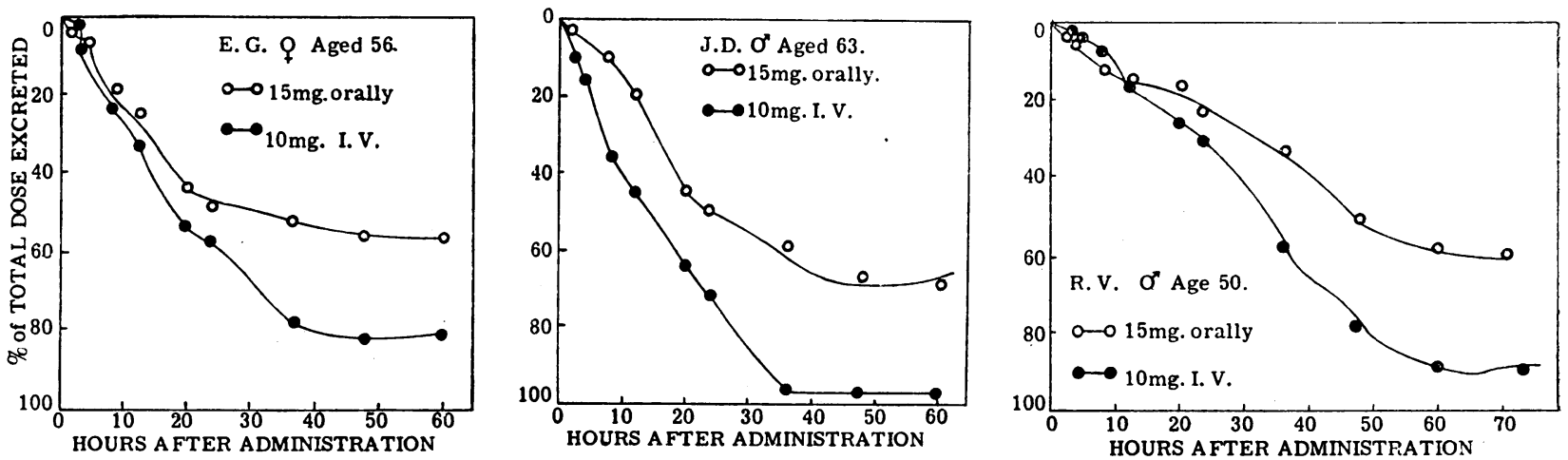

Fic. 1.-Rate of urinary excretion of mecamylamine following oral and intravenous administration of the drug to three subjects.

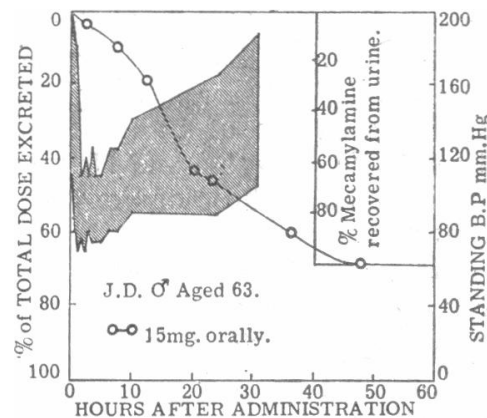

Fig. 2.-Comparison of rate of urinary excretion of mecamylamine with its hypotensive action folluwing oral administration in two subjects.

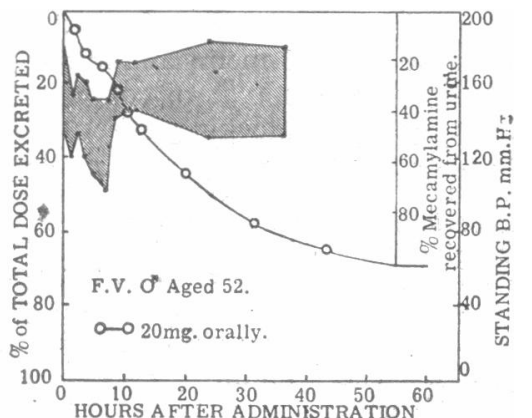
during ordinary maintenance therapy. The administration, however, of alkalis and acetazolamide may seriously interfere with excretion and might produce severe hypotension as well as other side-effects.

\section{Recovery of Mecamylamine from the Urine During Maintenance Therapy}

The 24-hour excretion of mecamylamine in three patients receiving continuous therapy was investigated and the results are shown in Fig 5. The drug was administered at the same times each day, and the 24-hour collection of urine was begun and ended at 8 a.m. In all cases the urinary recovery during the first $\mathbf{2 4}$ hours after starting mecamylamine was low, but the excretion thereafter increased to a reasonably steady level. If the first three days of treatment are excluded, the recovery of mecamylamine during

recovered in the first 24 hours if the urine is acid. That the production of an alkaline tension consequent upon the retention mecamylamine within the body is illustrated Fig. 4. no observations were made here on its effect in human subjects. Since the urine is there would be any risk of a sudden accumulation of mecamylamine in the body due to variations in urinary reactions
Fig. 2 compares the amount of mecamylamine excreted in the urine with the effect on the blood pressure following an oral dose. The fall in blood pressure has been further compared with the percentage of the total amount recovered from the urine. It can be seen that there is a significant fall of pressure within 30 minutes of administration, but the maximum effect does not occur until after one to two hours. mately $10-20 \%$ less after oral administration than after ence increases to $20-30 \%$. The total recovery after a single dose intravenously was between 80 and $98 \%$; after an oral dose it was approximately $65 \%$.

\section{Hypotensive Effect of Mecamylamine Compared with Urinary Excretion}


24 hours varied between 46 and $86 \%$ of the oral dose, the average being $65 \%$. During these observations no measurements of the urinary $p \mathrm{H}$ were made, but no patients received alkalis or acetazolamide whilst being treated with mecamylamine. In view of the fact that human urine is usually acid in reaction, it is not thought that the variations observed were due to any alterations in $p \mathrm{H}$. If it can be assumed that the excretion of mecamylamine reflects its absorption, then these findings suggest that it is fairly consistently and reliably absorbed during continued oral administration.

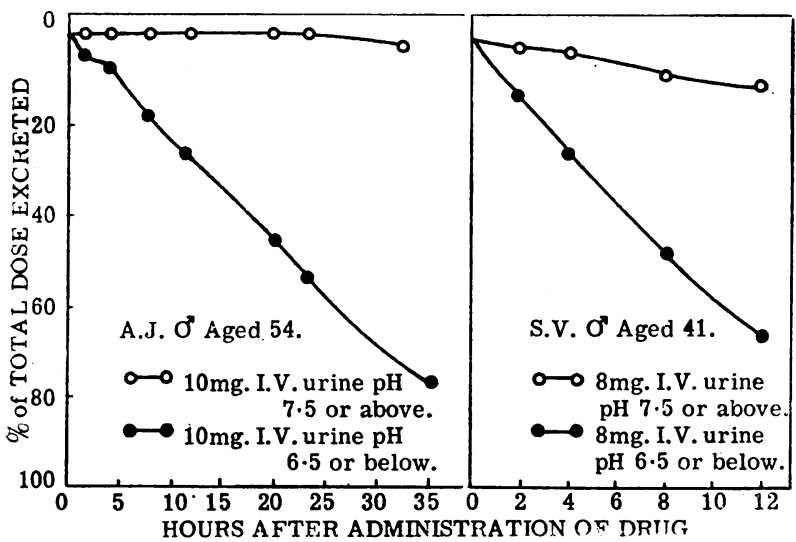

Fig. 3.-Effect of urinary $p \mathrm{H}$ on excretion of mecamylamine following a single intravenous dose in two subjects.

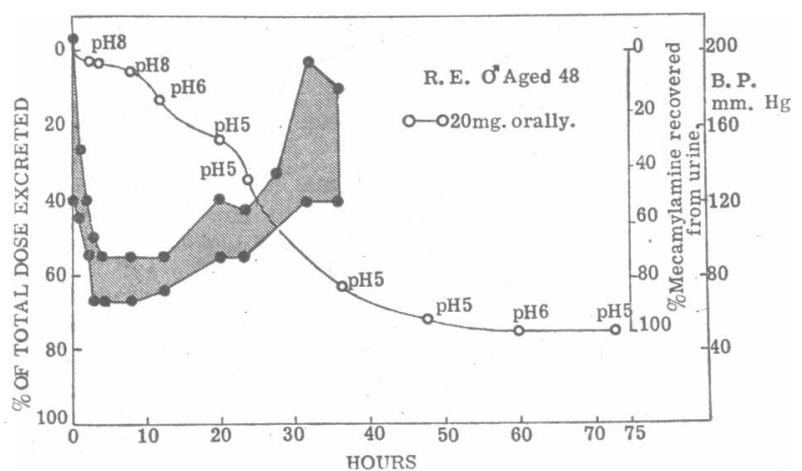

Fig. 4.-Effect of urinary $p \mathrm{H}$ on excretion of mecamylamine and duration of hypotension following a single oral dose of $20 \mathrm{mg}$.

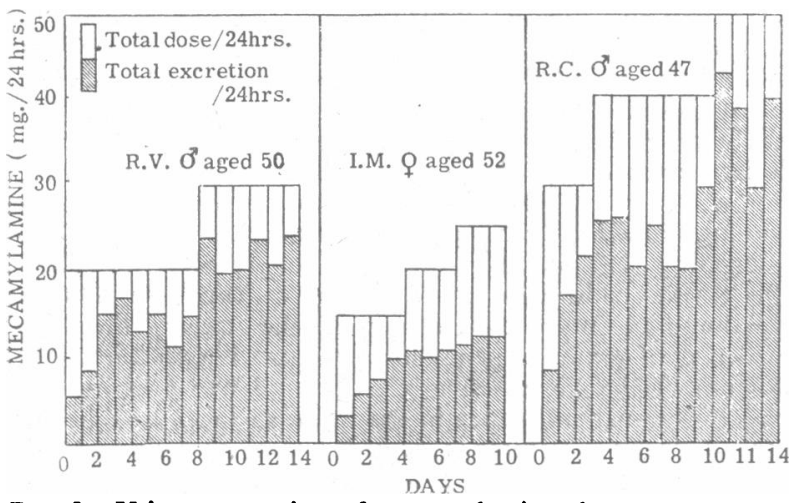

FIG. 5.-Urinary excretion of mecamylamine shown as mg. per 24 hours and as percentage of the daily oral dose in three subjects followed from beginning of treatment.

\section{Conclusions}

These observations upon the excretion and duration of effect of mecamylamine suggest that it possesses at least some of the pharmacological properties desirable in a hypotensive agent for oral administration. Unlike hexamethonium and pentolinium, a large proportion, between 55 and $75 \%$, of an oral dose may be recovered from the urine, which seems to indicate satisfactory absorption from the alimentary tract. Excretion is steady and prolonged, approximately $50 \%$ or less of the total amount recovered after a single dose appearing in the urine during the first 12 hours after administration. Likewise, there is a smooth and sustained fall of blood pressure following an oral dose. Daily excretion studies have suggested that mecamylamine is consistently and reliably absorbed within reasonably narrow limits. In the light of these findings blood-pressure controls should be obtained with relatively infrequent oral dosage and the blood pressure should not be subject to the fluctuation which has made treatment with other ganglion-blocking agents somewhat unsatisfactory. Doyle et al. (1956) have already published a report on the use of mecamylamine in 45 patients, and state that adequate control may be achieved with two doses daily. If the urine is alkaline, excretion of mecamylamine is almost entirely prevented, and it is likely that administration of acetazolamide had a similar effect. In either circumstance accumulation of the drug may occur and a prolonged fall of blood pressure will result.

We should like to thank Dr. K. S. MacLean, assistant director of the department of medicine, Guy's Hospital, for his helpful advice and encouragement, and for allowing us to make observations upon his patients. We are indebted to Messrs. Merck, Sharp and Dohme for generous supplies of mecamylamine (inversine).

\section{REFERENCES}

Baer, J. E., Paulson, S. F., Russo, H. F., and Beyer, K. H. (1956). Amer. J. Physiol., 186, 180

Brodie, B. B., and Udenfriend, S. (1945). J. biol. Chem., 158, 705

Doyle, A. E., Murphy, E. A., and Neilson, G. H. (1956). Brit. med. J. 2, 1209.

and Smirk, F. H. (1955). Practitioner, 174, 135.

Ford, R. V., Madison, J. C., and Moyer, J. H. (1956). Amer. J. med. Sci., 232, 129.

Freis, E. D. (1955). Lancet, 2, 977

Harington, M. (1953). Clin. Sci., 12, 185.

McMichael, J., and Murphy. E. A. (1955). J. chron. Dis., 1, 527.

Smirk F. H.

\section{Medical Memoranda}

\section{T. violaceum Infection of the Scalp}

Trichophyton violaceum infections are uncommon in this country. Walker (1950), in an account of the cultural findings of 2,015 cases of tinea capitis seen in the U.K. over a three-year period, mentions three children all of whom caught the infection abroad. There were also two adult cases of tinea circinata. Whittle (1956) adds another case, and again the child was infected abroad. Recently, however, more cases have occurred, although unreported in the literature (Walker, 1956, personal communication).

Sagher (1947), in Jerusalem, found that $56 \%$ of cultures from infected hairs were $T$. violaceum. He quotes figures showing that the disease is common in Hungary, Italy, Russia, and Japan, but rare in Western Europe. Miedzinski and Lipski (1956) also showed that $42 \%$ of Polish scalp ringworm was due to this fungus. In America the infection appears to be commoner in Texas (Lehmann, Pipkin, and Ressmann, 1950) than in New York, where Franks and Taschdjian (1956) isolated it only 9 times in 2,800 cultures.

Most clinical accounts-for example, Berlin and Meyrovitz (1955)-are those of "black dot" ringworm, but Walker (1950) mentions kerion formation. The following cases are therefore of interest because of the diversity of the clinical findings and the mode of infection.

\section{CASE RePorts}

Case 1.- In June, 1956, I was asked to see the 8-year-old daughter of a local minister. She had a crusted eruption of the vertex of the scalp of five weeks' duration, and also a small vesicular lesion on the back of the right hand. 